\title{
Feeder-Free Derivation of Functional Platelets from Human Induced Pluripotent Stem Cells
}

Pei-Ju Sung ${ }^{1}$, Sayandip Mukherjee ${ }^{1}$, Michael P Blundell ${ }^{1}$ and Adrian J Thrasher ${ }^{1 *}$

${ }^{1}$ Centre for Immunodeficiency, UCL Institute of Child Health, 30 Guilford Street, WC1N 1EH, London, UK

\begin{abstract}
Derivation of patient specific induced pluripotent stem cells, in combination with directed platelet differentiation, provides a promising platform to study human thrombopoiesis or platelet disorders, and has clear potential for future clinical applications. However, efficient differentiation of human induced pluripotent stem cells (iPSCs) into functional blood cells remains challenging. In this study, we report a novel protocol for the generation of CD41a+, $\mathrm{CD} 42 \mathrm{~b}+$, and $\mathrm{CD} 61+$ functional platelets from human iPSCs under feeder-free culture conditions. Platelets derived from this feeder-free system showed similar fibrinogen binding activity after agonist stimulation when compared to platelets derived from a previously reported cell line co culture system. Evidence showed these culture-derived platelets responded to different agonist stimulation by increased expression of activation markers (CD62P and PAC1) as expected. Together, these results provide an important step towards generating in vitro functional platelets from an unlimited source of patient-specific induced pluripotent stem cells using an animal component-free culture system.
\end{abstract}

Keywords: Human induced pluripotent stem cells; Platelets; Haematopoietic differentiation; Feeder-free cultures; Embryoid bodies (EBs)

\section{Introduction}

Since the successful derivation of induced pluripotent stem cells (iPSC) from somatic differentiated cells [1,2], iPSCs have provided exciting prospects in regenerative medicine [3]. Attempts have been carried out to differentiate human embryonic stem cells (hESCs) or human induced pluripotent stem cells (hiPSCs) into fully functional mature blood cells. Two different strategies have been widely employed for generating hematopoietic progenitors from hESCs or hiPSCs and further differentiating them into different hematopoietic lineages: (1) feeder-dependent coculture system with animal stromal-derived cell lines [4-8]; (2) feeder-free culture systems using embryoid bodies (EBs) [9-12] or serum-free monolayer cultures [13]. In the first differentiation protocol based on animal-derived stromal cells (e.g. OP9 or MS5 cells), human ES or iPS cells have been successfully differentiated into myeloid, lymphoid, erythroid, or megakaryocytic lineage cells with variable efficiencies $[4,14,15]$. In the second method, feeder-free EBbased protocol provides an animal component-free culture condition to generate hematopoietic progenitors, which is more amenable for adoption for future clinical applications in terms of in vitro generation of hematopoietic cells.

Platelets are derived from megakaryocytes (MKs). Approximately 2,000-10,000 platelets can be produced from each megakaryocyte precursor cell [16].Cytokines such as thrombopoietin (TPO), interleukin (IL)-3, IL-6, IL-9, IL-11, bone morphogenetic protein (BMP)-4, flt3 ligand (Flt-3L), and stem cell factor (SCF) are thought to facilitate MK maturation and platelet production. During maturation, large polyploid MKs (up to $64 \mathrm{~N}$ in DNA content) and proplatelets are formed, and platelets are released from proplatelet processes into the blood $[17,18]$. Platelet production involves the acquisition of characteristic cell surface markers such as CD41a, CD42a, CD42b, CD42d, and CD61. Expression of CD41 and CD42 on platelets is associated with CD61 in a complex to form the integrin $\alpha \operatorname{IIb} \beta 3$, which plays a major role to form mature and functional platelets [19].

Successful in vitro generation of mature MKs or platelets has been achieved from CD34+ hematopoietic stem cells derived from human bone marrow, cord blood and peripheral blood by culturing in medium containing cytokines such as TPO, SCF, IL-6 or IL-11 [20-26]. Additionally, to differentiate human ES/iPS cells into MKs or platelets, protocols have been published using either continuous OP9 coculture methods [27-29] or EB-based methods to initially generate hematopoietic progenitors followed by a period of coculture with OP9 cells [30]. However, use of animal-derived stromal cells presents a major hurdle in the adoption of these protocols for clinical evaluation. Therefore, in this study, we developed an animal component-free protocol for facilitating the release and collection of functional platelets from hiPSC-derived cultures. This protocol comprises two major steps: (i) induction of mesodermal hematopoietic progenitors by EB formation in mesodermal induction medium containing hBMP4 and other cytokines for hematopoietic progenitor differentiation, (ii) expansion and direct differentiation of hematopoietic progenitors into platelets in platelet differentiation medium.

\section{Methods}

\section{Ethics statement}

All animals were maintained in strict accordance with good animal practice as defined by UK Home Office Animal Welfare Legislation. All animal work in this study was approved by the Institutional Research Ethics Committee (Institute of Child Health, University College London, UK). All of the human samples were obtained after approval from the Ethical Review Board of the Institute of Child Health/Great Ormond Street Hospital and after obtaining written informed consent from subjects.

*Corresponding author: Adrian J Thrasher, Centre for Immunodeficiency, UCL Institute of Child Health, 30 Guilford Street, WC1N 1EH, London, UK. Tel: 0044 207813 8490; E-mail: a.thrasher@ucl.ac.uk

Received June 13, 2013; Accepted August 07, 2013; Published August 09, 2013

Citation: Sung PJ, Mukherjee S, Blundell MP, Thrasher AJ (2013) Feeder-Free Derivation of Functional Platelets from Human Induced Pluripotent Stem Cells. J Blood Disorders Transf 4:153. doi:10.4172/2155-9864.1000153

Copyright: $\odot$ 2013Sung PJ, et al. This is an open-access article distributed under the terms of the Creative Commons Attribution License, which permits unrestricted use, distribution, and reproduction in any medium, provided the original author and source are credited. 


\section{Generation of human induced pluripotent stem cells (hiPSCs)}

Human iPSCs were established from normal fibroblasts using a single excisable polycistronic lentiviral vector previously described by Sommer et al., and provided kindly by Dr. Gustavo Mostoslavsky, Boston University [31,32]. All cell cultures were maintained in a humidified incubator with $5 \% \mathrm{CO}_{2}$ at $37^{\circ} \mathrm{C}$. Fibroblasts were seeded in a 6-well plate one day before transduction. Cells were infected with polycistronic vector virus on day 0 . Two days post-infection, culture medium was replaced with human reprogramming medium - mTeSR $^{\circ} 1$ supplemented with $10 \mu \mathrm{M}$ Rock inhibitor, and two small molecules (2 $\mu \mathrm{M}$ of ALK5 inhibitor SB413542 (Stemgent) and $0.5 \mu \mathrm{M}$ MEK inhibitor PD0325901 (Stemgent)) [33]. The media were changed every other day until the induced ES-like colonies were picked up at 21 days postinfection for fibroblasts. The individual ES-like clones were expanded in mTeSR 1 medium (Stem cell technologies) and media were changed every day. Cre-mediated excision of reprogramming vector was performed by employing a non-integrating lentiviral vector expressing Cre recombinase. To maintain the undifferentiated status, iPS cells were passaged as clumps with TrypLE ${ }^{\mathrm{m} m}$ express (Life technologies Ltd) when they reached approximately 70-80\% confluence, usually every 5-7 days with a slit ratio about 1:3-1:6, and transferred to Matrigel ${ }^{\mathrm{TM}}$ (BD Biosciences)-coated plates with mTeSR1 growth medium.

\section{Immunofluorescent staining of human iPSCs}

For immunofluorescent staining, hiPSCs were fixed in $4 \%$ paraformaldehyde for 10 minutes at room temperature. After washing with PBS, cells were then incubated with PBS containing 3\% BSA and $0.1 \%$ Triton X-100. Primary antibodies (dilution in 1:000) used in this study were SSEA4, Oct4, TRA-1-60, TRA-1-81 (all from Millipore), c-myc, and Nanog (Abcam), and. For detection of primary antibodies, fluorophore-labeled (Alexa fluor 488 or 568; Molecular Probes) secondary antibodies were used according to the manufacturer's instructions.

\section{Extraction of RNA for RT-PCR}

Total RNA was extracted by using Qiagen RNeasy Kit (Qiagen). cDNA was synthesized using a Multiscribe Reverse Transcriptase kit according to the instructions supplied by the manufacturer (Applied Biosystems). PCR was performed with gene-specific primers.

\section{Teratoma formation assay}

Approximately $2 \times 10^{6}$ cells suspended in $150 \mathrm{ul}$ of RPMI containing Matrigel $^{\mathrm{TM}}$ (BD Biosciences) were injected subcutaneously in immunodeficient mice (RAG2 $2^{--}, \mathrm{\gamma c}^{-/}, \mathrm{C}^{--}$). Eight to ten weeks after injection, the tumours were dissected and fixed in $4 \%$ paraformaldehyde, embedded in paraffin, sectioned, stained with hematoxylin and eosin, and also processed for immunohistochemistry.

\section{Differentiation of hiPSCs into platelets}

To obtain platelets, we followed a two-step differentiation protocol adapted from Lu et al. with modifications [30]. In the first step, hiPSCs were induced to give rise to mesodermal hematopoietic progenitors, followed by their final differentiation into platelets. Briefly, on day 0 hiPSCs were dissociated with Dispase (Stemcell Technologies) at $37^{\circ} \mathrm{C}$ for 7 minutes, and approximately $2 \times 10^{5}$ cells $/ \mathrm{ml}$ of hiPSCs were placed on ultra-low attachment 6-well plates (Corning) in serumfree Stemline medium (Sigma-Aldrich) supplemented with BMP4 (50 ng/ml; PeproTech), VEGF (50 ng/ml; PeproTech), and bFGF (20 $\mathrm{ng} / \mathrm{ml}$; PeproTech) and cultured for 2 days. On day 2, fresh medium containing the same concentrations of BMP4, VEGF, and bFGF, plus
SCF, TPO, and FLT3 ligand ( $20 \mathrm{ng} / \mathrm{ml}$ each; PeproTech) was added to the cells for 6 days. Differentiation media were changed every 3 days and embryoid body (EB) formation was observed during this process. On day 8 of differentiation, differentiated cultures were collected for FACS measurements of CD34 expression, and the rest of cells were transferred to a fresh ultra-low attachment 6-well plate in platelet differentiation medium which consisted of serum-free Stemline medium (Sigma-Aldrich) with the addition of $50 \mathrm{ng} / \mathrm{ml} \mathrm{TPO,} 20 \mathrm{ng} /$ $\mathrm{ml} \mathrm{SCF}$, and $20 \mathrm{ng} / \mathrm{ml} \mathrm{IL-11} \mathrm{(all} \mathrm{from} \mathrm{PeproTech)} \mathrm{for} 6$ days. On day 14 of differentiation, cells were collected for FACS analysis and the rest of the cells were plated again in platelet differentiation medium $(100 \mathrm{ng} /$ $\mathrm{ml} \mathrm{TPO}, 50 \mathrm{ng} / \mathrm{ml} \mathrm{SCF}, 20 \mathrm{ng} / \mathrm{ml} \mathrm{IL}-11$ ) on an ultra-low attachment plate for an additional 8 days of feeder-free culture condition. GM6001 (100uM, Sigma-Aldrich) was added to the feeder-free cultures at the later stage of differentiation. For OP9 cells coculture condition, the differentiated cells collected on day 14 were plated onto mitomycin $\mathrm{C}$ (100 ng/ml)-treated OP9 stromal cells, with OP9 growth medium plus $100 \mathrm{ng} / \mathrm{ml}$ TPO (PeproTech), $50 \mathrm{ng} / \mathrm{ml} \mathrm{SCF}$ (PeproTech), and $25 \mathrm{U} /$ $\mathrm{ml}$ sodium heparin (Sigma-Aldrich) for an additional 8 days. Platelets were collected for FACS analysis and functional assays on day 22 of differentiation.

\section{Flow cytometric analysis of human iPSC-derived hematopoietic progenitors and platelets}

In brief, floating and loosely adherent cells were harvested by gentle pipetting, washed and spun at $200 \mathrm{~g}$ at $4^{\circ} \mathrm{C}$ to remove the large cells. Supernatant was collected for a further spin at $900 \mathrm{~g}$ for 10 minutes to obtain the platelet pellet. Fluorochrome-conjugated antibodies for lineage markers, CD34 (BD Biosciences), CD41, CD42, and CD61 (Ebioscience, Ltd.) were used to detect lineage cells in dilutions as recommended by the manufacturer. Cells were washed and stained with appropriate antibodies for 1 hour on ice. The cells were acquired on a FACS CyAn ${ }^{\text {mu }}$ ADP and analysed using Summit v4.3 software.

\section{In vitro functional analysis of hiPSC-derived platelets}

Human iPSC-derived platelets were collected and centrifuged at $150 \mathrm{~g}$ for 10 minutes to eliminate any large cells. The supernatant was centrifuged again at $400 \mathrm{~g}$ for 10 minutes to obtain platelet pellet. Washed platelets were incubated at $37^{\circ} \mathrm{C}$ for $0.5-1$ hour before any functional assays were performed. For functional studies of platelets, cells were stimulated by platelet agonists -thrombin $(1 \mathrm{U} /$ $\mathrm{ml}$ ), Adenosine diphosphate (ADP, $50 \mu \mathrm{M}$ ), and thrombin receptoractivating polypeptides (AYPGKF peptide, $500 \mu \mathrm{M}$, all from SigmaAldrich), incubated for 20 minutes at room temperature. Cells were washed and stained with anti-human CD41, CD42, CD61 (Ebioscience, Ltd), FITC-conjugated CD62P (BD Biosciences), and FITC-conjugated PAC1 (BD Biosciences) antibodies. The binding of CD62P (P-selectin) and PAC1 was quantified using flow cytometry. For controls, platelets were incubated with fluorescence-labeled antibodies without agonist stimulation.

\section{Isolation of human blood platelets}

Approximately $25 \mathrm{ml}$ of human blood was collected in a Vacutainer tube containing acid citrate. Whole blood was centrifuged immediately at $200 \mathrm{x}$ g for 15 minutes at room temperature to obtain platelet-rich plasma. 0.5 $\mu \mathrm{M}$ PGE1 was added to the platelet-rich plasma supernatant to avoid platelet activation, along with $1 / 10$ volume of sodium citrate anticoagulant. This was centrifuged at $700 \times \mathrm{g}$ for 10 minutes at room temperature. The platelet pellet was washed with HEPES-Tyrode buffer $\left(134 \mathrm{mM} \mathrm{NaCl}, 12 \mathrm{mM} \mathrm{NaHCO}{ }_{3}, 2.9 \mathrm{mM} \mathrm{KCl}, 0.34 \mathrm{mM} \mathrm{Na}_{2} \mathrm{HPO}_{4}, 1\right.$ 
$\mathrm{mM} \mathrm{MgCl}, 5 \mathrm{mM}$ glucose, $10 \mathrm{mM}$ Hepes, $\mathrm{pH} 7.4)$, and centrifuged at $800 \mathrm{x} \mathrm{g}$ for 5 minutes. Final suspensions of washed platelets were adjusted to $1 \times 10^{7}$ platelets $/ \mathrm{ml}$. Platelets were incubated at $37^{\circ} \mathrm{C}$ for 0.5 1 hour before functional assays were performed.

\section{FACS measurements of fibrinogen binding activity}

Fibrinogen binding activity was determined by flow cytometric analysis with the use of fluorescence-labeled anti-human fibrinogen (DAKO UK LTD). Human iPSC-derived platelets and human blood platelets were stimulated with platelet agonists (ADP- $50 \mu \mathrm{M}$; Thrombin-1 U/ml; AYPGKF peptide- $500 \mu \mathrm{M}$ ) in the presence of human fibrinogen or CD61 antibody for 30 minutes at $37^{\circ} \mathrm{C}$ without stirring. The mixtures were then diluted and washed with HEPES buffer for flow cytometric analysis. Control cells were incubated with fluorescence-labeled antibodies without platelet agonist stimulation.

\section{Results}

\section{Generation and characterization of human iPSCs}

Human skin fibroblasts were cultured to passage 1-4 before reprogramming using a cre-exicisable polycistronic lentiviral vector encoding Oct-4, Sox-2, Klf-4, and c-myc [31,32]. All isolated iPS clones showed typical human ES cell-like morphology and were positive for alkaline phosphatase activity (Figure 1A).Two iPSC lines were used for the following studies. Non-integrating vector mediated cre-excision was carried out to remove the reprogramming transgenes, and RT-PCR was employed to confirm no exogeneous expression of four transcription factors after cre excision (Figure 1B). Immuno fluorescence staining revealed that after cre excision the iPS colonies expressed different pluripotent stem cell markers TRA-1-60, SSEA-4, Oct-4, Nanog, and TRA-1-81 (Figure 1C).To determine differentiation potential in vivo, hiPSCs were injected subcutaneously into immunodeficient mice and observed for tumour growth at the site of injection.Histological analysis of haematoxylin/eosin-stained tumour sections and immunostaining revealed the presence of ectodermal, mesodermal, and endodermal structures (Figure 1D). Thus, iPS cells were able to spontaneously differentiate into derivatives of all three germ layers in vivo.

\section{Derivation of platelets from hiPSCs}

In this study, we first generated platelets from human iPS cells using a feeder-free culture system. We established a defined two-stage culture protocol for generating CD34+ hematopoietic progenitors and further differentiated them into platelets using a differentiation medium containing a combination of cytokines required for megakaryopoiesis. The differentiation protocol is schematically represented in Figure 2AD. All results represented here are from more than three independent experiments using two different human iPSC lines. In the initial induction step (Figure 2A), hiPSCs were maintained in a lowattachment feeder-free condition in the presence of BMP4, VEGF, and bFGF for 2 days to form cellular aggregates known as embryoid bodies (EBs). EBs was transferred to a hematopoietic differentiation medium containing BMP4, VEGF, bFGF, SCF, TPO, and Flt-3L for 6 days. Flow cytometry analysis at the end of this initial haematopoietic induction phase (day 8 ) revealed $10 \%$ of population was CD34+ hematopoietic progenitors (Figure $2 \mathrm{~B}$ ). Next, the entire population was cultured in platelet differentiation medium containing TPO, SCF and IL11 for a duration of 6 days. On day 14 of differentiation, immune phenotyping results showed a population consisted of CD61+ precursors (Figure 2C). CD61 is a marker for human platelets and their precursors, and is widely used for the detection of megakaryoblastic differentiation. Few CD41a+ and CD42b+ cells were observed at this stage. Different
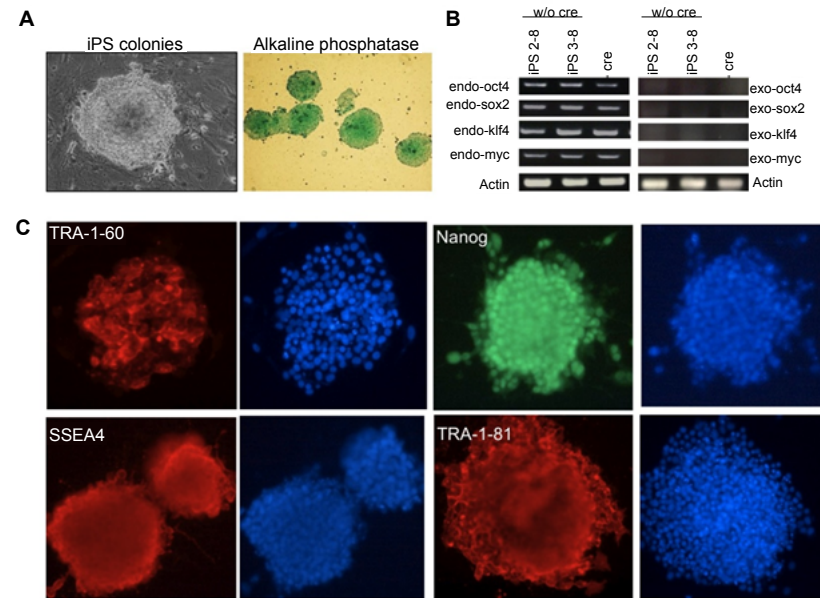

Actin
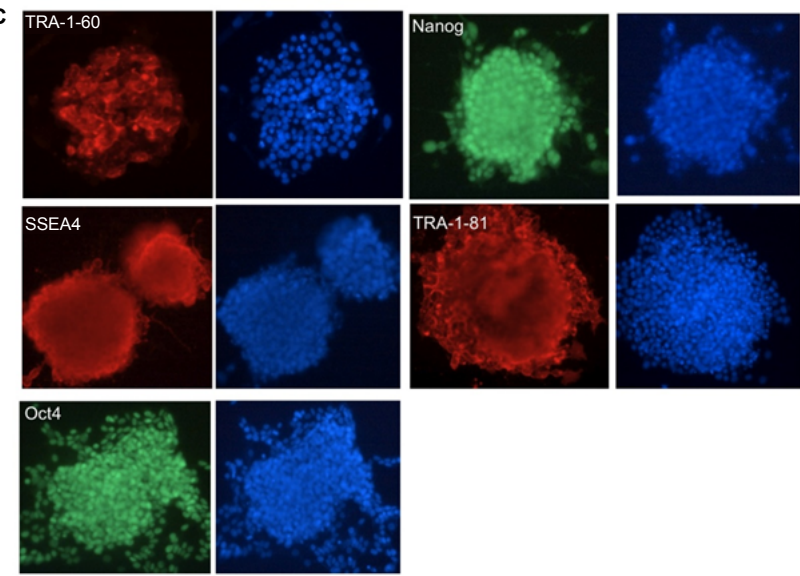

D
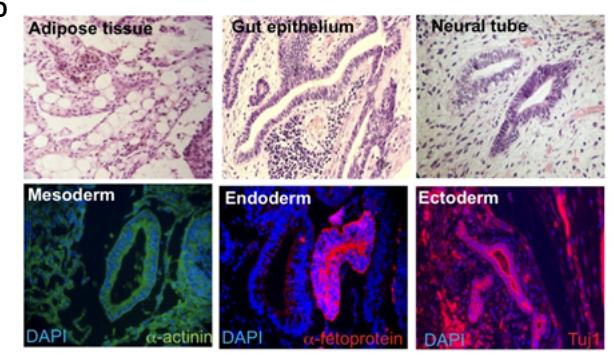

Figure 1: Induction of human iPSCs after reprogramming by combining transgene Oct4, Klf4, Sox2, and C-Myc. (A) Images of iPS colonies and positive staining for alkaline phosphatase. (B) Expression levels of endogenous and exogenous OCT4, SOX2, Klf4, and c-myc (relative to Actin) assessed by RTPCR. (C) Staining of iPS colonies was positive for a panel of pluripotent markers: TRA-1-60, SSEA-4, Oct4, Nanog, TRA-1-81 expression. (D) Hematoxylin and eosin staining showed teratoma formation from hiPS cells containing multiple tissues and positively immunostaining for $\alpha$-actin (mesoderm marker), $\alpha$-fetoprotein (endoderm marker), and $\beta$-tubulin (ectoderm marker).

replicates were derived from independently reprogrammed iPSC lines.

We subjected the cultures on day14 to two different conditions for an additional 8 days of differentiation: (i) continued feeder-free culture condition in platelet differentiation medium supplemental with high levels of TPO, SCF, and IL11; (ii) OP9 coculture condition maintained in a special platelet differentiation medium (described in Materials and Methods). To determine whether platelets were generated from both culture conditions, cells released to the culture medium on day 22 of differentiation (end of differentiation protocol) were analyzed by flow cytometry to detect expression of platelet markers CD41a, CD42b, and CD61.Using platelets derived from human peripheral blood, a gate was fixed based on forward- and side-scatter to establish proper size gating for platelets derived from hiPSCs (Figure 2D). When the same gate was applied to differentiation cultures, the majority of the cells collected from the differentiation medium localized within this gate. Consistent with previous reports showing a size discrepancy between in vitro generated human platelets as compared with human blood platelets $[28,30]$, we also found that hiPSC-platelets were more heterogeneous 
Citation: Sung PJ, Mukherjee S, Blundell MP, Thrasher AJ (2013) Feeder-Free Derivation of Functional Platelets from Human Induced Pluripotent Stem Cells. J Blood Disorders Transf 4:153. doi:10.4172/2155-9864.1000153

than human blood platelets. This platelet gate was used for the following FACS analysis of hiPSC-derived platelets.

As platelet differentiation continued until day 22, expression of $\mathrm{CD} 41 \mathrm{a}$ and $\mathrm{CD} 42 \mathrm{~b}$ reached a considerable level while expression of CD61 decreased in both culture conditions (Figure 2E). Expression of CD41a, CD42b and CD61 decreased after day 22 (data not shown). Cells obtained from day 22 developed a population of CD41a+ and $\mathrm{CD} 42 \mathrm{~b}+$ cells. The percentage of CD41+ cells increased from $2.6 \%$ to $18.2 \pm 5.62 \%$ in feeder-freeculture condition, and $15.5 \pm 4.9 \%$ in OP9 coculture condition; CD $42 \mathrm{~b}+$ cells increased from $2.2 \%$ to $28.8 \pm 1 \%$ in feeder-free culture condition and $11.2 \pm 6.4 \%$ in OP9 coculture condition. CD61+ proportion decreased from $75.5 \pm 3.3 \%$ to $33.7 \pm$
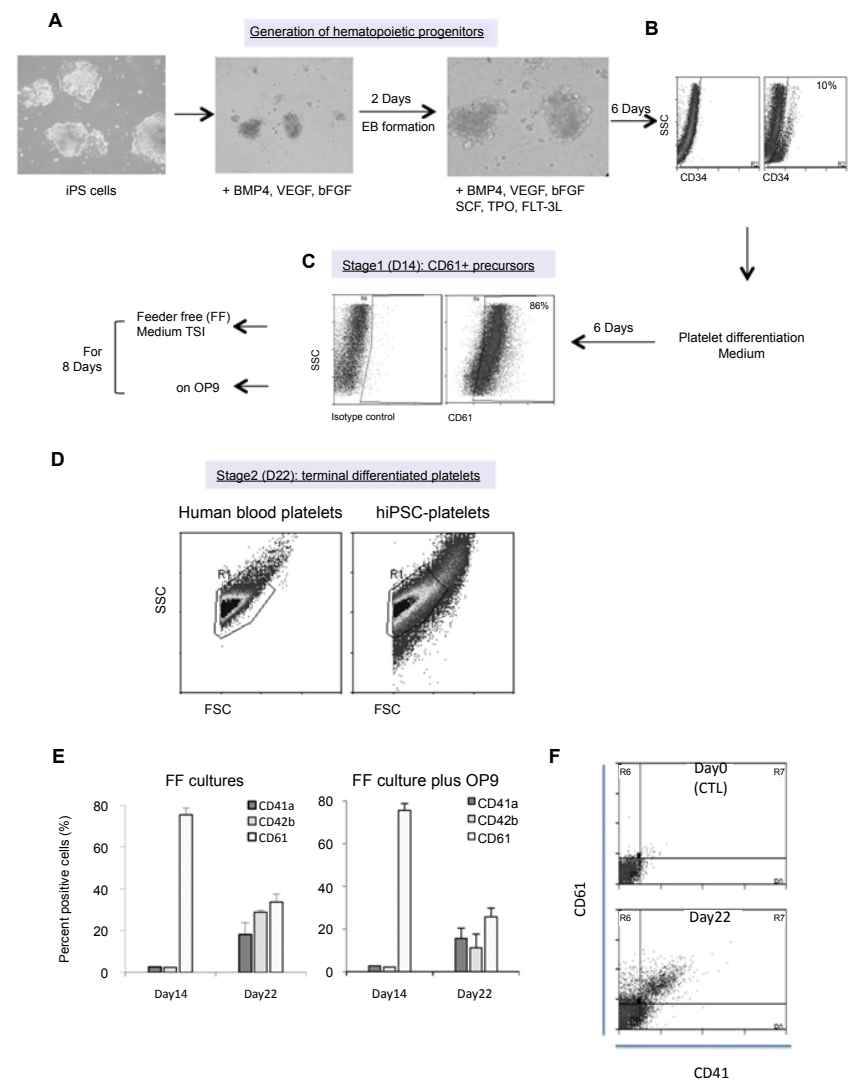

Figure 2: Schematic diagram of the protocol used to directly differentiate human iPSCs into functional platelets. (A) Undifferentiated hiPS cells cultured in StemLine hematopoietic stem cell expansion medium plus hBMP4, VEGF, and bFGF for 2 days, and SCF, TPO, and FIt-3L were added for another 6 days to generate hematopoietic progenitors. (B) On day 8 of differentiation, flow cytometric analysis showed the generation of hiPSC-derived CD34+ hematopoietic progenitors. (C) After culturing in platelet differentiation medium for 6 days, a population of CD61+ progenitorswas obtained. Cells were collected and grown on OP9 feeder cells or continuously grown in platelet differentiation medium for an additional 8 days. (D) Cells released to the culture medium on day 22 of differentiation (end of differentiation) were analysed by flow cytometry. A platelet gate was fixed in the forward- and side-scatter profiles of human blood platelets (left panel) to establish proper size gating for platelets derived from iPSCs. Majority of the cells collected from the differentiation medium localized in this gate. This platelet gate was used for the following FACS analysis of culture-derived platelets. (E) Percentage of platelet marke expression (CD41a, CD42b, and CD61) on day14 and day 22 of differentiation derived from feeder-free (FF) culture system and OP9 coculture system.(F) Coexpression of CD41 and CD61 was found on Day22 of differentiation. All data represented here are from more than three independent experiments using two different human iPSC lines.
$3.86 \%$ in feeder-free culture condition, and $25.6 \pm 4.2 \%$ in OP9 co culture condition. The results showed that cells collected from feederfree culture condition exhibited slightly higher percentage of CD41a, $\mathrm{CD} 42 \mathrm{~b}$, and CD61 positive cells when compared to the cells derived from OP9 co culture condition. As shown in Figure 2F, compared to the non-differentiated control, co-expression of CD41 and CD61 was found on Day22.

\section{Functional analysis of iPSC-derived platelets}

Platelets obtained from day 22 were subjected to in vitro functional assays. The differentiation protocol was repeated more than 5 times, obtaining platelets from independent experiments using two different human iPSC lines. We first tested the response of hiPSC-derived platelets to agonist stimulation. Platelet agonist stimulation induces conformational changes of integrin $\alpha \operatorname{IIb} \beta 3$ and results in cluster formation, which promotes the binding of fibrinogen to activated platelets [34,35]. FACS measurements of fibrinogen binding activity were examined to compare the hiPSC-platelets derived from feederfree cultures and OP9 cocultures following platelet agonist stimulation with ADP. Similar levels of fibrinogen binding activity (50-60\%) were detected in hiPSC-platelets derived from feeder-free and OP9 cultures (Figure 3A). The results suggested that platelets derived from feederfree and OP9 coculture system expressed similar levels of platelet markers (CD41a, CD42b, and CD61) and also achieved the same level of activation in response to agonist stimulation. A feeder-free culture protocol to generate platelets is preferable because it eliminates the contamination of animal components from murine stromal cells (OP9 cells). Therefore, we used hiPSC-platelets derived from feeder-free condition to perform a variety of in vitro functional assays.

To explore in vitro functionality of hiPSC-platelets derived from feeder-free cultures, flow cytometric measurements of $\alpha \operatorname{IIb} \beta 3$ activation were performed following platelet agonist stimulation. Platelets derived from feeder-free conditions were obtained from more than three independent experiments for functionality analysis. Three platelet agonists (ADP, thrombin, and a thrombin receptoractivating polypeptide (AYPGKF)) were used separately in this study to activate the culture-derived platelets. PAC-1 and CD62P were used to measure the activation after platelet agonist stimulation. Increased binding of CD62P was observed in activated hiPSC-platelets following treatment with platelet agonists (ADP, thrombin, or AYPGKF peptide), as compared to the resting controls (Figure 3B, left panel).Surface expression of PAC-1 was also observed after agonist stimulation, but not in unstimulated controls (Figure 3B, right panel).

\section{Comparison of iPSC-derived platelets and human blood platelets}

When using forward- and side-scatter histogram of human peripheral blood platelets to establish proper size gating for hiPSCplatelets, approximately $99 \%$ of human blood platelets were $\mathrm{CD} 42 \mathrm{~b}+$ and $\mathrm{CD} 61+$, and $12 \%$ of hiPSC-platelets were CD42b+ and CD61+ (Figure $4 \mathrm{~A}$ for human blood platelets, and 4B for iPSC-platelets). On activation, platelet $\alpha \operatorname{IIb} \beta 3$ integrin binding to fibrinogen stimulates thrombus formation [35]. Therefore, to determine the extent of functional $\alpha \operatorname{IIb} \beta 3$ integrin expression that occurred during activation of hiPSC-platelets or human blood platelets, FACS measurements of fibrinogen binding assay were performed on activated platelets after agonist stimulation. In response to agonist stimulation (ADP, thrombin, or AYPGKF peptide), a shift of fibrinogen binding activity was observed from both human blood platelets and hiPSC-derived platelets (Figure 4C). More than 
A

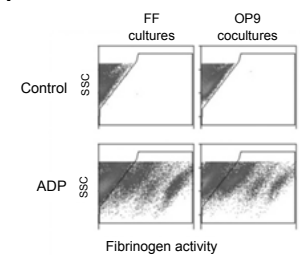

B

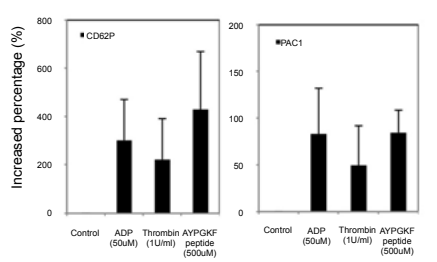

Figure 3: Functional characterization of hiPSC-platelets in vitro. (A) The four panels are representative dot plots for iPSC-platelets derived from feeder-free (FF) cultures or OP9 cultures binding to fibrinogen antibody in the absence (top) and presence (bottom) of ADP (50uM). Platelets derived from both FF and OP9 cultures show similar fibrinogen binding activity in response to platelet agonist stimulation. (B) FACS analysis of agonist-dependent surface marker expression of CD62P and PAC-1 upon different platelet agonists (e.g. ADP. Thrombin, AYPGKF peptide) from hiPSC-platelets derived from feeder-free culture condition. Increase binding of CD62P and PAC-1 was observed from activated platelets after agonist stimulation. All data represented here are from more than three independent experiments using two different human iPSC lines.

$80 \%$ of hiPSC-platelets were detected to bind to fibrinogen after agonist stimulation. In addition, dramatic binding activity of fibrinogen and CD61 to activated human platelets was detected (about 99\%, in Figure 4D); while moderate binding activity (ranging from $50-64 \%$ according to different agonists, in Figure 4E) was detected from activated hiPSC-platelets in response to platelet agonists. We found fibrinogen and CD61 binding to activated hiPSC-platelets was weaker than the binding to activated human blood platelets, suggesting hiPSC-platelets maybe less mature than human blood platelets. Consistent with a previous report of generating platelets from human ES cells, culturederived platelets exhibited less binding capacity after stimulation when compared to human blood platelets [30]. Many factors may contribute to this disparity, for example, the main reason could be heterogeneous distribution of differentiation cultures, resulting in less maturity of hiPSC-platelets compared to human blood platelets. We also observed most of human blood platelets were CD42b+/CD61+, while only part of the differentiation cultures were CD42b+/CD61+ platelets. The undifferentiated or partially-differentiated particles may also contribute to the decreased binding capacity. Taken together, the results of fibrinogen binding assay indicated that platelets derived from hiPSCs were functional in vitro and comparable to the platelets isolated from human peripheral blood.

\section{Discussion}

Platelets are a nucleate and the smallest cells in body $(2-3 \mu \mathrm{m}$ in diameter) - less than one-third the diameter of red blood cells. They play an essential role in hemostasis and thrombosis, and also contribute to inflammation, repair of vascular injury, embryonic development, immunologic responses, and tumour angiogenesis or metastasis [36,37]. There is a high demand for transfusable platelets in patients with thrombocytopenia for multiple clinical applications. There has therefore been considerable interest in generating functional platelets from renewable sources including human ES cells [28-30] and human iPSCs [27]. The ability to generate functional platelets in vitro from a potentially unlimited and autologous source of cells, such as hiPSC, could provide significant therapeutic advantages. In the present study, we demonstrated for the first time that human iPSCderived platelets can be successfully generated from defined feederfree culture conditions. Human iPSC-derived platelets generated from

this protocol were functionally comparable to human platelets isolated from peripheral blood. This feeder-free methodology constitutes an important first steptowards the ultimate goal of clinically applicable platelet production from pluripotent stem cells.

Several systems have been established to promote in vitro hematopoietic differentiation of human ES/iPS cells. Growth of human pluripotent cells in suspension culture in the presence of various combinations of cytokines and growth factors (e.g. Flt-3L, VEGF, BMP4, SCF, G-CSF, IL3, IL6, TPO etc), leads to differentiation towards different hematopoietic lineages $[9,11,38,39]$. During mega karyocytopoiesis and thrombocytopoiesis, TPO (thrombopoietin) is the principal cytokine that enhances MK development. It has been
A

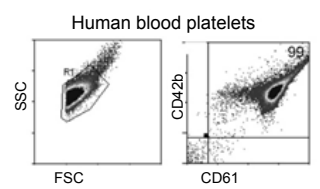

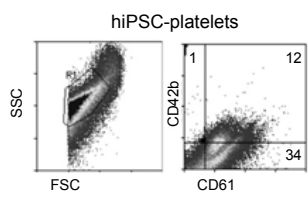

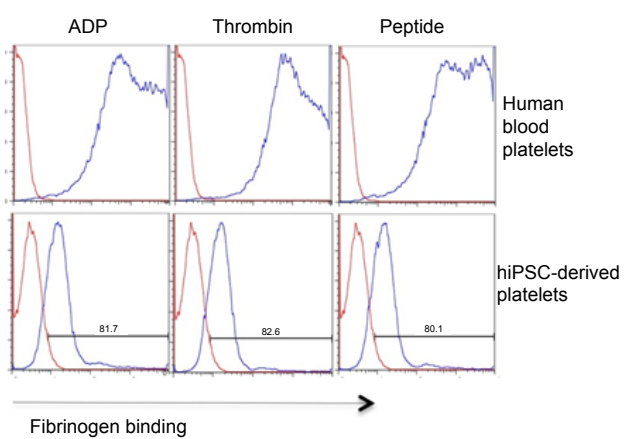

D

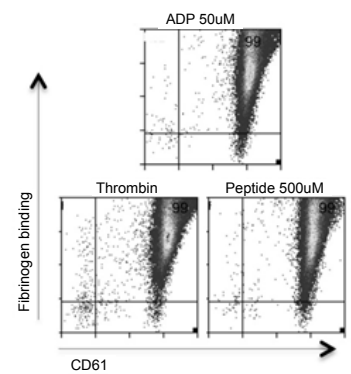

$\mathbf{E}$

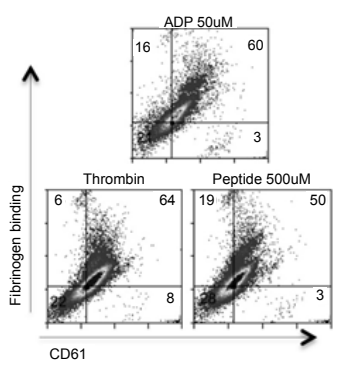

Figure 4: Comparison of platelets generated from human iPS cells (hiPSCplatelets) and human peripheral blood platelets. (A) A platelet gate was fixed based on the forward- and side-scatter profiles of human peripheral blood platelets (left). 99\% of the gated particles were CD42b+ and CD61+ (right) from human blood platelets. (B) The two panels show a flow cytometry FSC vs SSC profile (left) from hiPSC-derived platelets using the same platelet gate. Approximately $12 \%$ of hiPSC-PLTs expressed both CD42b and CD61. (C-E) FACS measurements of fibrinogen binding activity of hiPSC-derived platelets compared to human blood platelets: (C) Comparison of fibrinogen binding shifts upon different platelet agonist stimulation from hiPSC-derived platelets and human platelets. (D) In human blood platelets: A dramatic binding of fibrinogen and CD61 to activated platelets was observed after agonist stimulation. (E) In hiPSC-derived platelets: A moderate binding of fibrinogen and CD61 to activated hiPSC-platelets was observed after agonist stimulation.The FACS results represented here were chosen from three independent experiments. 
Citation: Sung PJ, Mukherjee S, Blundell MP, Thrasher AJ (2013) Feeder-Free Derivation of Functional Platelets from Human Induced Pluripotent Stem Cells. J Blood Disorders Transf 4:153. doi:10.4172/2155-9864.1000153

widely used to enrich $\mathrm{MK}$ populations from CD34+ cells derived from various sources such as peripheral blood stem cells, bone marrow, and umbilical cord blood [21-23,26]. Previous studies have indicated that combinations of cytokines such as TPO, SCF and IL6 or IL11 enhance MK development and maturation. In our study, we generated hematopoietic progenitors from human iPSC lines based on EB formation using a cytokine cocktail containing BMP4, VEGF, bFGF, SCF, TPO, and Flt-3L. We directly differentiated these hematopoietic progenitors towards the megakaryocytic lineage in differentiation medium containing TPO, SCF and IL11. The threedimensional spherical EB structure was maintained throughout all the stages of differentiation. FACS analysis suggested that platelet particles expressing CD41a, CD42b, and CD61 were successfully generated from hiPS cells after 22 days of differentiation.

Mature MKs or platelets have been successfully generated from mouse ES cells uponco-culturing with murine stromal cell lines $[18,40,41]$. Recent studies have also demonstrated the feasibility of the generation of human MKs or platelets from hESCs/hiPSCs based on OP9 coculture protocols $[27,28,30]$. Lu et al. reported generation of platelets in a feeder-free system from human ESC although these appeared to exhibit less functionality than those generated from a more conventional OP9 coculture system [30]. To develop an animal component-free system, we amended Lu's protocol to differentiate hiPSCs into platelets using EB-based feeder-free culture conditions and further examined the response of platelets upon stimulation. Similar to platelets generated under OP9 coculture conditions, we found platelets generated from feeder-free conditions showed equivalent levels of fibrinogen binding activity, although this was somewhat reduced compared with blood-derived human platelets indicating a level of immaturity.

This study provides an important first step to generate an autologous source of platelets from human iPS cells without the use of animal stromal cell lines. The platelet yield was approximately $10^{7}$ from $10^{4}$ hiPS cells (data not shown). Further improvement of methodology will therefore be required to generate sufficient numbers of plateletsfor in vivo functional assays and for clinical applications. Employing genemanipulated ESCs or disease-specific iPSCs, in combination with effective differentiation protocols, will also be useful for in vitro models of disease pathology, thereby providing further insights into normal thrombopoiesis and human platelet disorders.

\section{Acknowledgements}

We thank Dr Wei-Li Di for generously providing human skin fibroblasts. This work was supported by European Union Funding PERSIST-FP7. AJT is supported by the Wellcome Trust and Great Ormond Street Hospital Children Charity. The authors report no conflicts of interest.

\section{References}

1. Takahashi K, Tanabe K, Ohnuki M, Narita M, Ichisaka T, et al. (2007) Induction of pluripotent stem cells from adult human fibroblasts by defined factors. Cell 131: 861-872.

2. Takahashi K, Yamanaka S (2006) Induction of pluripotent stem cells from mouse embryonic and adult fibroblast cultures by defined factors. Cell 126 663-676.

3. Park IH, Arora N, Huo H, Maherali N, Ahfeldt T, et al. (2008) Disease-specific induced pluripotent stem cells. Cell 134: 877-886.

4. Choi KD, Yu J, Smuga-Otto K, Salvagiotto G, Rehrauer W, et al. (2009) Hematopoietic and endothelial differentiation of human induced pluripotent stem cells. Stem Cells 27: 559-567.

5. Slukvin II, Vodyanik MA, Thomson JA, Gumenyuk ME, Choi KD (2006) Directed differentiation of human embryonic stem cells into functional dendritic cells through the myeloid pathway. J Immunol 176: 2924-2932.
6. Lieber JG, Webb S, Suratt BT, Young SK, Johnson GL, et al. (2004) The in vitro production and characterization of neutrophils from embryonic stem cells. Blood 103: 852-859.

7. Vodyanik MA, Bork JA, Thomson JA, Slukvin II (2005) Human embryonic stem cell-derived CD34+ cells: efficient production in the coculture with OP9 stromal cells and analysis of lymphohematopoietic potential. Blood 105: 617-626.

8. Hill KL, Kaufman DS (2008) Hematopoietic differentiation of human embryonic stem cells by cocultivation with stromal layers. Curr Protoc Stem Cell Biol Chapter 1: Unit 1F.

9. Ng ES, Davis RP, Hatzistavrou T, Stanley EG, Elefanty AG (2008) Directed differentiation of human embryonic stem cells as spin embryoid bodies and a description of the hematopoietic blast colony forming assay. Curr Protoc Stem Cell Biol Chapter 1: Unit 1D.

10. Lengerke C, Grauer M, Niebuhr NI, Riedt T, Kanz L, et al. (2009) Hematopoietic development from human induced pluripotent stem cells. Ann N Y Acad Sc 1176: 219-227.

11. Cerdan C, Hong SH, Bhatia M (2007) Formation and hematopoietic differentiation of human embryoid bodies by suspension and hanging drop cultures. Curr Protoc Stem Cell Biol Chapter 1: Unit 1D.

12. Kennedy M, D'Souza SL, Lynch-Kattman M, Schwantz S, Keller G (2007) Development of the hemangioblast defines the onset of hematopoiesis in human ES cell differentiation cultures. Blood 109: 2679-2687.

13. Niwa A, Heike T, Umeda K, Oshima K, Kato I, et al. (2011) A novel serum-free monolayer culture for orderly hematopoietic differentiation of human pluripotent cells via mesodermal progenitors. PLoS One 6: e22261.

14. Choi KD, Vodyanik MA, Slukvin II (2009) Generation of mature human myelomonocytic cells through expansion and differentiation of pluripotent stem cell-derived lin-CD34+CD43+CD45+ progenitors. J Clin Invest 119: 2818-2829.

15. Choi KD, Vodyanik M, Slukvin II (2011) Hematopoietic differentiation and production of mature myeloid cells from human pluripotent stem cells. Nat Protoc 6: 296-313.

16. Long MW (1998) Megakaryocyte differentiation events. Semin Hematol 35 192-199.

17. Kosaki G (2005) In vivo platelet production from mature megakaryocytes: does platelet release occur via proplatelets? Int J Hematol 81: 208-219.

18. Fujimoto TT, Kohata S, Suzuki H, Miyazaki H, Fujimura K (2003) Production of functional platelets by differentiated embryonic stem (ES) cells in vitro. Blood 102: $4044-4051$.

19. Phillips DR, Charo IF, Parise LV, Fitzgerald LA (1988) The platelet membrane glycoprotein Ilb-IIla complex. Blood 71: 831-843.

20. Reems JA, Pineault N, Sun S (2010) In vitro megakaryocyte production and platelet biogenesis: state of the art. Transfus Med Rev 24: 33-43.

21. Choi ES, Nichol JL, Hokom MM, Hornkohl AC, Hunt P (1995) Platelets generated in vitro from proplatelet-displaying human megakaryocytes are functional. Blood 85: 402-413.

22. Norol F, Vitrat N, Cramer E, Guichard J, Burstein SA, et al. (1998) Effects of cytokines on platelet production from blood and marrow CD34+ cells. Blood 91: 830-843.

23. Sun L, Tan P, Yap C, Hwang W, Koh LP, et al. (2004) In vitro biological characteristics of human cord blood-derived megakaryocytes. Ann Acad Med Singapore 33: $570-575$

24. Ungerer M, Peluso M, Gillitzer A, Massberg S, Heinzmann U, et al. (2004) Generation of functional culture-derived platelets from CD34+ progenitor cells to study transgenes in the platelet environment. Circ Res 95: e36-44.

25. Matsunaga T, Tanaka I, Kobune M, Kawano Y, Tanaka M, et al. (2006) Ex vivo large-scale generation of human platelets from cord blood CD34+ cells. Stem Cells 24: $2877-2887$

26. Gandhi MJ, Drachman JG, Reems JA, Thorning D, Lannutti BJ (2005) A nove strategy for generating platelet-like fragments from megakaryocytic cell lines and human progenitor cells. Blood Cells Mol Dis 35: 70-73.

27. Takayama N, Nishimura S, Nakamura S, Shimizu T, Ohnishi R, et al. (2010) Transient activation of c-MYC expression is critical for efficient platele generation from human induced pluripotent stem cells. J Exp Med 207: 2817 
Citation: Sung PJ, Mukherjee S, Blundell MP, Thrasher AJ (2013) Feeder-Free Derivation of Functional Platelets from Human Induced Pluripotent Stem Cells. J Blood Disorders Transf 4:153. doi:10.4172/2155-9864.1000153

Page 7 of 6

28. Takayama N, Nishikii H, Usui J, Tsukui H, Sawaguchi A, et al. (2008) Generation of functional platelets from human embryonic stem cells in vitro via ES-sacs VEGF-promoted structures that concentrate hematopoietic progenitors. Blood 111: 5298-5306.

29. Gaur M, Kamata T, Wang S, Moran B, Shattil SJ, et al. (2006) Megakaryocytes derived from human embryonic stem cells: a genetically tractable system to study megakaryocytopoiesis and integrin function. J Thromb Haemost 4: 436-442.

30. Lu SJ, Li F, Yin H, Feng Q, Kimbrel EA, et al. (2011) Platelets generated from human embryonic stem cells are functional in vitro and in the microcirculation of living mice. Cell Res 21: 530-545.

31. Sommer CA, Sommer AG, Longmire TA, Christodoulou C, Thomas DD, et al. (2010) Excision of reprogramming transgenes improves the differentiation potential of iPS cells generated with a single excisable vector. Stem Cells 28 : 64-74.

32. Sommer CA, Stadtfeld M, Murphy GJ, Hochedlinger K, Kotton DN, et al. (2009) Induced pluripotent stem cell generation using a single lentiviral stem cell cassette. Stem Cells 27: 543-549.

33. Lin T, Ambasudhan R, Yuan X, Li W, Hilcove S, et al. (2009) A chemical platform for improved induction of human iPSCs. Nat Methods 6: 805-808.

34. Wagner DD, Burger PC (2003) Platelets in inflammation and thrombosis. Arterioscler Thromb Vasc Biol 23: 2131-2137.
35. Shattil SJ, Newman PJ (2004) Integrins: dynamic scaffolds for adhesion and signaling in platelets. Blood 104: 1606-1615.

36. Broos K, Feys HB, De Meyer SF, Vanhoorelbeke K, Deckmyn H (2011) Platelets at work in primary hemostasis. Blood Rev 25: 155-167.

37. Leslie M (2010) Cell biology. Beyond clotting: the powers of platelets. Science 328: $562-564$.

38. Pick M, Azzola L, Mossman A, Stanley EG, Elefanty AG (2007) Differentiation of human embryonic stem cells in serum-free medium reveals distinct roles for bone morphogenetic protein 4, vascular endothelial growth factor, stem cell factor, and fibroblast growth factor 2 in hematopoiesis. Stem Cells 25: 22062214.

39. Dravid GG, Crooks GM (2011) The challenges and promises of blood engineered from human pluripotent stem cells. Adv Drug Deliv Rev 63: 331 341.

40. Tamaru S, Kitajima K, Nakano T, Eto K, Yazaki A, et al. (2008) Calyculin A retraction of mature megakaryocytes proplatelets from embryonic stem cells. Biochem Biophys Res Commun 366: 763-768.

41. Eto K, Murphy R, Kerrigan SW, Bertoni A, Stuhlmann H, et al. (2002) Megakaryocytes derived from embryonic stem cells implicate CalDAG-GEFI in integrin signaling. Proc Natl Acad Sci U S A 99: 12819-12824.
Citation: Sung PJ, Mukherjee S, Blundell MP, Thrasher AJ (2013) Feeder-Free Derivation of Functional Platelets from Human Induced Pluripotent Stem Cells. J Blood Disorders Transf 4:153. doi:10.4172/2155-9864.1000153 